\title{
THERMALLY STIMULATED DEPOLARIZATION CURRENT IN HETEROGENEOUS STRUCTURES. THE DIELECTRIC MATRIX WITH DIELECTRIC SPHERES
}

\author{
J. PoźNIAK \\ Institute of Low Temperature and Structure Research, Polish Academy of Sciences \\ P.O. Box 937, 50-950 Wrocław, Poland
}

(Received March 24, 1992)

\begin{abstract}
Theoretical analysis of the thermally stimulated depolarization current in heterogeneous structure is performed. Two systems are considered: (i) a single dielectric sphere in dielectric matrix and (ii) a continuous dielectric medium containing sparsely distributed dielectric spheres (Wagner-Voigt model of heterogeneous dielectric. The existence of the interfacial charge is taken into account. The equations are found which describe the dependence of the thermally stimulated depolarization current density on the polarization conditions and on the electrical properties of the both materials.
\end{abstract}

PACS numbers: $77.30 .+d$

\section{Introduction}

A considerable number of theories has been elaborated and more or less successfully applied to explain the relaxation effects induced by the action of an external alternating electric field on some heterogeneous systems (e.g. [1-5]). They are based on the assumption that the characteristic material parameters, like conductivity and dielectric permittivity, appearing in pertinent relations can be treated as constants. In reality, the values of these parameters are temperature-dependent. Hence, the above theories can be applied to describe the isothermal effects only.

Theoretical approach to the relaxation processes appearing in homogeneous dielectrics at changing temperature, like field-induced thermally stimulated depolarization (TSD) and polarization (TSP) currents, was presented in [4-10]. In these considerations, the material parameters were treated as temperature-dependent variables.

A simplified theory of TSP and TSD currents in heterogeneous dielectric systems, in which the charge carriers are accumulated at interfaces of the components 
(Maxwell-Wagner effect), has been proposed only by Harašta and Thurzo [11] and by Van Turnhout [4]. The basic assumption of this theory is that the properties of heterogeneous dielectric are the same as the properties of the parallel-plate two-layer condenser (Maxwell model) with well-defined thicknesses.

In the real heterogeneous systems the particles of one dielectric are sparsely distributed in another dielectric. For this reason, in the present paper an attempt is undertaken to perform calculations of the TSD current density for the system consisting of dielectric matrix filled with sparsely distributed dielectric spheres (Wagner-Voigt model).

\section{Theory}

\subsection{Single dielectric sphere in dielectric matrix}

We shall consider a model of a heterogeneous system consisting of a single dielectric sphere of radius $R$, conductivity $\kappa_{2}$ and dielectric constant $\varepsilon_{2}$, located in dielectric matrix with conductivity $\kappa_{1}$ and dielectric constant $\varepsilon_{1}$.

During the first step of TSD measurement the sample is polarized in a constant electric field $E_{0}$ at a constant temperature $T_{\mathrm{p}}[6-10]$. Hence, in order to relate TSD current to the polarization conditions we must consider firstly the polarization process itself. For this purpose we take the centre of the sphere as the origin of the spherical coordination system $(r, \theta, \Psi)$. We choose the $z$-axis in the direction of the electric field $E_{0}$ and assume that the angle $\theta$ is equal to zero in this direction.

Under these conditions the potential $V(t)$ outside the sphere satisfies the Laplace's equation

$$
\Delta V(t)=\nabla^{2} V(t)=0
$$

because of lack of any free space charge. On the surface of the sphere the Laplace's equation is not valid, because of accumulation of the surface charge (Maxwell-Wagner effect). Inside the sphere, however, the Laplace's equation can be used again. For this reason, for the description of $V(t)$ we must use two different functions $V_{1}(t)$ and $V_{2}(t)$, outside and inside the sphere, respectively.

The appropriate boundary conditions are:

(i) $\left.V_{1}(t)\right|_{r=R}=\left.V_{2}(t)\right|_{r=R}$, since the potentials must be equal on both sides of the interface.

(ii) Far from the sphere, the potential is determined only by the external electric field $E_{0}$ :

$$
\left.V_{1}(t)\right|_{r \rightarrow \infty}=-E_{0} r \cos \theta .
$$

(iii) The normal components of the dielectric displacements must be continuous at the boundary $(r=R)$ :

$$
\varepsilon_{0} \varepsilon_{1} \frac{\mathrm{d} E_{1}(t)}{\mathrm{d} t}+\kappa_{1} E_{1}(t)=\varepsilon_{0} \varepsilon_{2} \frac{\mathrm{d} E_{2}(t)}{\mathrm{d} t}+\kappa_{2} E_{2}(t),
$$

where $\varepsilon_{0}$ is the permittivity of the free space.

(iv) At the centre of the sphere $V_{2}(t)$ must not have a singularity. 
In the case of symmetry about the $z$-axis we can seek the solution to the problem in the form of a series

$$
\begin{aligned}
& V_{1}(t)=\sum_{n=0}^{\infty}\left[A_{n}(t) r^{n}+\frac{B_{n}(t)}{r^{n+1}}\right] P_{n}(\cos \theta), \\
& V_{2}(t)=\sum_{n=0}^{\infty}\left[C_{n}(t) r^{n}+\frac{D_{n}(t)}{r^{n+1}}\right] P_{n}(\cos \theta),
\end{aligned}
$$

where $P_{n}(\cos \theta)$ is the $n$-th Legendre polynomial.

From the above-mentioned boundary conditions it follows that

$$
A_{n}=B_{n}=C_{n}=D_{n}=0
$$

for all values of $n$ except for $n=1$. When $n=1$, we have

$$
V_{1}(t)=\left[A_{1}(t) r+\frac{B_{1}(t)}{r^{2}}\right] \cos \theta
$$

and

$$
V_{2}(t)=\left[C_{1}(t) r+\frac{D_{1}(t)}{r^{2}}\right] \cos \theta
$$

Next, applying the second and fourth boundary conditions to these solutions, we have

$$
A_{1}=-E_{0} \text { and } D_{1}=0 .
$$

Therefore

$$
\begin{aligned}
& V_{1}(t)=\left[\frac{B_{1}(t)}{r^{2}}-E_{0} r\right] \cos \theta \\
& V_{2}(t)=C_{1}(t) r \cos \theta
\end{aligned}
$$

The corresponding radial components of the electric fields are

$$
E_{1 r}(t)=-\frac{\partial V_{1}(t)}{\partial r}=\left[2 \frac{B_{1}(t)}{r^{3}}+E_{0}\right] \cos \theta,
$$

and

$$
E_{2 r}(t)=-\frac{\partial V_{2}(t)}{\partial r}=-C_{1}(t) \cos \theta
$$

Moreover, on account of the first boundary condition and the solutions (2) we obtain

$$
C_{1}(t)=\frac{B_{1}(t)}{R^{3}}-E_{0}
$$

Introduction of Eqs. (3) to the third boundary condition yields the differential equation

$$
\frac{\mathrm{d} B_{1}(t)}{\mathrm{d} t}+\frac{1}{\tau} B_{1}(t)=E_{0} R^{3} \frac{\kappa_{2}-\kappa_{1}}{\varepsilon_{0}\left(2 \varepsilon_{1}+\varepsilon_{2}\right)}
$$


where

$$
\tau^{-1}=\frac{2 \kappa_{1}+\kappa_{2}}{\varepsilon_{0}\left(2 \varepsilon_{1}+\varepsilon_{2}\right)}
$$

The solution of differential Eq. (4) is

$$
B_{1}(t)=B_{1}(\infty)+\left[B_{1}(0)-B_{1}(\infty)\right] \exp (-t / \tau) .
$$

Consequently, we can obtain also

$$
C_{1}(t)=\left\{B_{1}(\infty)+\left[B_{1}(0)-B_{1}(\infty)\right] \exp (-t / \tau)\right\} R^{-3}-E_{0} .
$$

For the relation between the two electric fields and the density of the interface charges $q(t)$ we have from the Gauss law

$$
q(t)=\left.\varepsilon_{0} \varepsilon_{1} E_{1}(t)\right|_{r=R}-\left.\varepsilon_{0} \varepsilon_{2} E_{2}(t)\right|_{r=R} .
$$

The initial value $B_{1}(0)$ can be found from Eqs. (3), (6), (7), and (8) by taking into account that the initial value of the interfacial charge density $q(0)$ is equal to zero. Consequently, we obtain

$$
B_{1}(0)=\frac{\varepsilon_{2}-\varepsilon_{1}}{2 \varepsilon_{1}+\varepsilon_{2}} E_{0} R^{3} .
$$

Now, we will calculate the final value $B_{1}(\infty)$, i.e., the value of $B_{1}(t)$ when the system is in stationary state. In this case $\mathrm{d} E_{1}(t) / \mathrm{d} t=\mathrm{d} E_{2}(t) / \mathrm{d} t=0$ and the third boundary condition reduces to

$$
\left.\kappa_{1} E_{1}(t)\right|_{r=R}=\left.\kappa_{2} E_{2}(t)\right|_{r=R} .
$$

Performing calculations (similar to those given above) we obtain

$$
\begin{aligned}
& B_{1}(\infty)=\frac{\kappa_{2}-\kappa_{1}}{2 \kappa_{1}+\kappa_{2}} E_{0} R^{3}, \\
& C_{1}(\infty)=\frac{3 \kappa_{1}}{2 \kappa_{1}+\kappa_{2}} E_{0} .
\end{aligned}
$$

Consequently

$$
\begin{aligned}
& V_{1}(\infty)=-\left[1-\frac{\kappa_{2}-\kappa_{1}}{2 \kappa_{1}+\kappa_{2}}\left(\frac{R}{r}\right)^{3}\right] E_{0} r \cos \theta, \\
& V_{2}(\infty)=-\frac{3 \kappa_{1}}{2 \kappa_{1}+\kappa_{2}} E_{0} r \cos \theta
\end{aligned}
$$

From the last equations and the Gauss law (8) we obtain for the surface charge density

$$
q(\infty)=3 \frac{\varepsilon_{0}\left(\varepsilon_{1} \kappa_{2}-\varepsilon_{2} \kappa_{1}\right)}{2 \kappa_{1}+\kappa_{2}} E_{0} \cos \theta
$$

Equation (12) can be used, among others, to calculate the magnitude of the total surface charge $Q$ accumulated on the interface. If the surface charge density $q$ is constant, then $Q=q S$, where $S$ is the surface area. As in our case the charge 
distribution is not uniform $(q=\mathrm{d} Q / \mathrm{d} S \neq 0)$, the total surface charge must be written as

$$
Q=\int_{S} q \mathrm{~d} S
$$

On account of the expression (12) and the geometry of the considered system, we obtain that the total charge of one sign (i.e., the charge accumulated on one half of the sphere) is

$$
Q(\infty)=3 \pi E_{0} R^{2} \frac{\varepsilon_{0}\left(\varepsilon_{1} \kappa_{2}-\varepsilon_{2} \kappa_{1}\right)}{2 \kappa_{1}+\kappa_{2}} .
$$

We see that the magnitude of the accumulated interfacial charge increases linearly with the intensity of applied electric field and is proportional to the difference between the products of conductivities and dielectric constants of the both materials.

Substitution of $B_{1}(t)$ and $C_{1}(t)$ from Eqs. (6) and (7), as well as of $B_{1}(\infty)$ and $B_{1}(0)$ from Eqs. (9) and (10), into Eqs. (3) yields

$$
\begin{aligned}
& E_{1 r}(t)=\left[1+\frac{2\left(\kappa_{2}-\kappa_{1}\right)}{2 \kappa_{1}+\kappa_{2}}\left(\frac{R}{r}\right)^{3}\right. \\
& \left.+\frac{6\left(\kappa_{1} \varepsilon_{2}-\kappa_{2} \varepsilon_{1}\right)}{\left(2 \varepsilon_{1}+\varepsilon_{2}\right)\left(2 \kappa_{1}+\kappa_{2}\right)}\left(\frac{R}{r}\right)^{3} \exp (-t / \tau)\right] E_{0} \cos \theta
\end{aligned}
$$

and

$$
E_{2 r}(t)=\frac{3 \kappa_{1}}{2 \kappa_{1}+\kappa_{2}}\left[1-\frac{\kappa_{1} \varepsilon_{2}-\kappa_{2} \varepsilon_{1}}{\kappa_{1}\left(2 \varepsilon_{1}+\varepsilon_{2}\right)} \exp (-t / \tau)\right] E_{0} \cos \theta
$$

According to the Gauss law (8) and the last expressions the time dependence of the interfacial charge density is given by

$$
q(t)=3 \frac{\varepsilon_{0}\left(\varepsilon_{1} \kappa_{2}-\varepsilon_{2} \kappa_{1}\right)}{2 \kappa_{1}+\kappa_{2}} E_{0} \cos \theta[1-\exp (-t / \tau)] .
$$

This equation can be used also to describe the polarization current density

$$
J(t)=\frac{\mathrm{d} q(t)}{\mathrm{d} t}=3 \frac{\kappa_{2} \varepsilon_{1}-\kappa_{1} \varepsilon_{2}}{2 \varepsilon_{1}+\varepsilon_{2}} E_{0} \cos \theta \exp (-t / \tau) .
$$

Combining Eqs. (8) and (13) we find that the total interfacial charge is the function of time given by

$$
Q(t)=3 \pi E_{0} R^{2} \frac{\varepsilon_{0}\left(\varepsilon_{1} \kappa_{2}-\kappa_{1} \varepsilon_{2}\right)}{2 \kappa_{1}+\kappa_{2}}[1-\exp (-t / \tau)] .
$$

Now, we can return to calculations of the TSD current in the considered system. During measurements of the TSD current the external voltage is switched off at a low temperature $T_{0}$ and the system is heated up at a constant rate $b=$ $\mathrm{d} T / \mathrm{d} t$, i.e., the temperature $T$ and time $t$ are linearly dependent variables

$$
T=T_{0}+b t .
$$

Hence, also in this case we can use (after introduction of a new variable $T$ ) the Laplace's equation to describe the potentials $V_{1}(T)$ and $V_{2}(T)$. We will seek the solutions of the Laplace's equation in the form of the series given by Eqs. (1), 
provided that the coefficients $A_{n}, B_{n}, C_{n}$, and $D_{n}$ are now treated as functions of temperature $T$.

The boundary conditions to be satisfied by $V(T)$ are as follows:

(v) $E_{0}=0$,

since during measurements of the TSD current the external field is switched off.

(vi) $\left.V_{1}(T)\right|_{r=R}=\left.V_{2}(T)\right|_{r=R}$,

since the function $V(T)$ is continuous across a boundary.

(vii) The normal components of the currents must be continuous at the interfacial boundary

$$
b \varepsilon_{0} \varepsilon_{1} \frac{\mathrm{d} E_{1}(T)}{\mathrm{d} T}+\kappa_{1}(T) E_{1}(T)=b \varepsilon_{0} \varepsilon_{2} \frac{\mathrm{d} E_{2}(T)}{\mathrm{d} T}+\kappa_{2}(T) E_{2}(T) .
$$

(viii) At the centre of the sphere the potential $V_{2}(T)$ must not have a singularity.

Taking into account the above-mentioned boundary conditions we find that $A_{n}, B_{n}, C_{n}$, and $D_{n}$ are equal to zero for all values of $n$ except for $n=1$. When $n=1$, we have

$$
A_{1}=D_{1}=0 \quad \text { and } \quad C_{1}=B_{1} / R^{3} .
$$

Consequently, we obtain that the corresponding radial components of the electric fields are

$$
\begin{aligned}
& E_{1 r}(T)=2 \frac{B_{1}(T)}{r^{3}} \cos \theta \\
& E_{2 r}(T)=-\frac{B_{1}(T)}{R^{3}} \cos \theta
\end{aligned}
$$

Introducing the expressions (17) to the boundary condition (vii) we find

$$
\frac{\mathrm{d} B_{1}(T)}{\mathrm{d} T}+\frac{1}{b \tau(T)} B_{1}(T)=0 .
$$

where

$$
\tau(T)^{-1}=\frac{2 \kappa_{1}(T)+\kappa_{2}(T)}{\varepsilon_{0}\left(2 \varepsilon_{1}+\varepsilon_{2}\right)} .
$$

Solution of this equation is

Consequently

$$
B_{1}(T)=B_{1}(0) \exp \left[-\frac{1}{b} \int_{T_{0}}^{T} \frac{\mathrm{d} T^{\prime}}{\tau\left(T^{\prime}\right)}\right] .
$$

$$
C_{1}(T)=\frac{B_{1}(0)}{R^{3}} \exp \left[-\frac{1}{b} \int_{T_{0}}^{T} \frac{\mathrm{d} T^{\prime}}{\tau\left(T^{\prime}\right)}\right] .
$$

Substitution of the $B_{1}(T)$ and $C_{1}(T)$ functions into Eqs. (17) gives

$$
E_{1 r}(T)=\frac{2 B_{1}(0)}{r^{3}} \cos \theta \exp \left[-\frac{1}{b} \int_{T_{0}}^{T} \frac{\mathrm{d} T^{\prime}}{\tau\left(T^{\prime}\right)}\right],
$$




$$
E_{2 r}(T)=-\frac{B_{1}(0)}{R^{3}} \cos \theta \exp \left[-\frac{1}{b} \int_{T_{0}}^{T} \frac{\mathrm{d} T^{\prime}}{\tau\left(T^{\prime}\right)}\right] .
$$

The initial value of $B_{1}(0)$ can be found from the Eqs. (19) and the Gauss law Hence

$$
q(0)=\left.\varepsilon_{0} \varepsilon_{1} E_{1 r}\left(T_{0}\right)\right|_{r=R}-\left.\varepsilon_{0} \varepsilon_{2} E_{2 r}\left(T_{0}\right)\right|_{r=R}
$$

$$
B_{1}(0)=\frac{R^{3} q(0)}{\varepsilon_{0}\left(2 \varepsilon_{1}+\varepsilon_{2}\right) \cos \theta} \equiv \alpha R^{3} .
$$

Now, we can assume that the initial value of charge density $q(0)$ is equal to the charge density acquired by the system during its polarization at the temperature $T_{\mathrm{p}}\left(T_{\mathrm{p}}>T_{0}\right)$, i.e., that it is given by Eq. (12). Therefore

$$
\alpha=\left.\frac{3 E_{0}}{2 \varepsilon_{1}+\varepsilon_{2}}\left(\frac{\varepsilon_{1} \kappa_{2}-\kappa_{1} \varepsilon_{2}}{2 \kappa_{1}+\kappa_{2}}\right)\right|_{T=T_{\mathrm{p}}} .
$$

Substitution of $B_{1}(0)$ into Eqs. (19) gives the temperature dependences of the radial components of the electric fields

and

$$
E_{1 r}(T)=2 \alpha\left(\frac{R}{r}\right)^{3} \cos \theta \exp \left[-\frac{1}{b} \int_{T_{0}}^{T} \frac{\mathrm{d} T^{\prime}}{\tau\left(T^{\prime}\right)}\right]
$$

$$
E_{2 r}(T)=-\alpha \cos \theta \exp \left[-\frac{1}{b} \int_{T_{0}}^{T} \frac{\mathrm{d} T^{\prime}}{\tau\left(T^{\prime}\right)}\right] .
$$

Consequently, taking into account the last equations, we obtain the following expression for the radial component of the local TSD current density:

$$
\begin{aligned}
& j(T)=j_{1}(T)=j_{2}(T)=\left.\frac{6 E_{0} \cos \theta}{\left(2 \varepsilon_{1}+\varepsilon_{2}\right)^{2}}\left(\frac{\varepsilon_{1} \kappa_{2}-\kappa_{1} \varepsilon_{2}}{2 \kappa_{1}+\kappa_{2}}\right)\right|_{T=T_{\mathbf{p}}} \\
& \times\left.\left(\kappa_{1} \varepsilon_{2}-\varepsilon_{1} \kappa_{2}\right)\right|_{T} \exp \left[-\frac{1}{b} \int_{T_{\mathbf{0}}}^{T} \frac{\mathrm{d} T^{\prime}}{\tau\left(T^{\prime}\right)}\right] .
\end{aligned}
$$

\subsection{System containing sparsely distributed dielectric spheres}

Let us consider now a model of a heterogeneous system consisting of $N$ dielectric spheres of radius $R$, conductivity $\kappa_{2}$ and dielectric constant $\varepsilon_{2}$, sparsely distributed in a dielectric matrix of conductivity $\kappa_{1}$ and dielectric constant $\varepsilon_{1}$. We restrict the considerations to the system in which the electric field around one sphere does not influence the field around the other spheres. This requirement implies that the distances between the neighbouring spheres must be large compared to their radii. At this condition [12] the heterogeneous system containing $N$ spheres of radius $R$ is equivalent to a single sphere of radius $R_{0}$, whose dielectric constant $\varepsilon$ and conductivity $\kappa$ are given by

$$
\varepsilon=\varepsilon_{1} \frac{\left(2 \varepsilon_{1}+\varepsilon_{2}\right)-2\left(\varepsilon_{1}-\varepsilon_{2}\right) v_{2}}{\left(2 \varepsilon_{1}+\varepsilon_{2}\right)+\left(\varepsilon_{1}-\varepsilon_{2}\right) v_{2}}
$$


and

$$
\kappa=\kappa_{1} \frac{\left(2 \kappa_{1}+\kappa_{2}\right)-2\left(\kappa_{1}-\kappa_{2}\right) v_{2}}{\left(2 \kappa_{1}+\kappa_{2}\right)+\left(\kappa_{1}-\kappa_{2}\right) v_{2}},
$$

where $v_{2}=N\left(R / R_{0}\right)^{3}$ is the volume fraction of the dispersed spheres. Consequently, the equations obtained for the single sphere can be also used for the system containing $N$ spheres. Obviously, these equations remain valid provided that $R$ is replaced by $R_{0}$ as well as the $\varepsilon_{2}$ and $\kappa_{2}$ are replaced by $\varepsilon$ and $\kappa$ given by Eqs. (21) and (22), respectively. In this way we obtain also the equations needed to correlate TSD current with the polarization conditions

$$
\begin{aligned}
& q(t)=\left.\frac{9 \varepsilon_{0} \varepsilon_{1} v_{2}}{\left(2 \varepsilon_{1}+\varepsilon_{2}\right)+\left(\varepsilon_{1}-\varepsilon_{2}\right) v_{2}}\left(\frac{\varepsilon_{1} \kappa_{2}-\kappa_{1} \varepsilon_{2}}{2 \kappa_{1}+\kappa_{2}}\right)\right|_{T=T_{\mathrm{P}}} \\
& \times E_{0} \cos \theta\left(1-\mathrm{e}^{-t / \tau}\right), \\
& Q(t)=9 \pi R^{2} \sqrt[3]{N^{2} v_{2}} \frac{\varepsilon_{0} \varepsilon_{1}}{\left(2 \varepsilon_{1}+\varepsilon_{2}\right)+\left(\varepsilon_{1}-\varepsilon_{2}\right) v_{2}} \\
& \times\left.\left(\frac{\varepsilon_{1} \kappa_{2}-\kappa_{1} \varepsilon_{2}}{2 \kappa_{1}+\kappa_{2}}\right)\right|_{T=T_{\mathrm{p}}} E_{0}\left(1-\mathrm{e}^{-t / \tau}\right),
\end{aligned}
$$

and

$$
j_{1}(t)=\frac{9 \kappa_{1} v_{2}\left(\varepsilon_{1} \kappa_{2}-\kappa_{1} \varepsilon_{2}\right)}{\left(2 \varepsilon_{1}+\varepsilon_{2}\right)\left[\left(2 \kappa_{1}+\kappa_{2}\right)+\left(\kappa_{1}-\kappa_{2}\right) v_{2}\right]} E_{0} \cos \theta \mathrm{e}^{-t / \tau}
$$

where

$$
\tau^{-1}=\frac{\kappa_{1}}{\varepsilon_{0} \varepsilon_{1}} \frac{\left(2 \kappa_{1}+\kappa_{2}\right)}{\left(2 \varepsilon_{1}+\varepsilon_{2}\right)} \frac{\left(2 \varepsilon_{1}+\varepsilon_{2}\right)+\left(\varepsilon_{1}-\varepsilon_{2}\right) v_{2}}{\left(2 \kappa_{1}+\kappa_{2}\right)+\left(\kappa_{1}-\kappa_{2}\right) v_{2}} .
$$

Finally, we can describe the density of the local TSD current by

$$
\begin{aligned}
& j(T)=\text { const } \cdot \frac{\kappa_{1}(T)\left[\kappa_{2}(T) \varepsilon_{1}-\kappa_{1}(T) \varepsilon_{2}\right]}{\left[2 \kappa_{1}(T)+\kappa_{2}(T)\right]+\left[\kappa_{1}(T)-\kappa_{2}(T)\right] v_{2}} \\
& \times \exp \left[-\frac{1}{b} \int_{T_{0}}^{T} \frac{\mathrm{d} T^{\prime}}{\tau\left(T^{\prime}\right)}\right]
\end{aligned}
$$

where

$$
\text { const }=\left.\frac{18 v_{2}^{2}\left(\kappa_{2} \varepsilon_{1}-\kappa_{1} \varepsilon_{2}\right)}{\left(2 \varepsilon_{1}+\varepsilon_{2}\right)^{2}\left(2 \kappa_{1}+\kappa_{2}\right)}\right|_{T=T_{\mathrm{p}}} E_{0} \cos \theta .
$$

Equation (27), similar to that describing TSD current arising from motion of electric dipoles [4-10], represents an asymmetrical "glow curve". In agreement with Eq. (28) the amplitude of this curve is dependent on the volume fraction of the dispered-phase particles and on the electric properties of the both materials at $T_{\mathrm{p}}$. It is also dependent on the polarization conditions, being a linear function of the polarizing field $E_{0}$. The fractional term in Eq. (27), which dominates in the low-temperature range, expresses the initial increase in the current with temperature. The exponential term in Eq. (27), which dominates at high temperature, describes the decay of the current. 


\section{Remarks}

It must be emphasized here that the charges, which are described by Eqs. (23) and (24), are imaginary, but yield the same potential in the given point of space as that due to the real charges accumulated on $N$ dielectric spheres of radius $R$. For the same reason we cannot use Eqs. (15) and (20) to calculate the local current density within any sphere of radius $R$.

Application of the present theory to experimental results and the comparison of the present and previous theories $[4,11]$ will be discussed in a separate paper.

\section{References}

[1] L.K.H. van Beek, in: Progress in Dielectric, Vol. 7, Ed. J.B. Birks, Heywood Books, London 1967, p. 69.

[2] J. Volger, in: Progress in Semiconductors, Vol. 4, Ed. A.F. Gibson, Heywood and Comp. Ltd., London 1960, p. 205.

[3] A.K. Jonscher, Dielectric Relaxation in Solids, Chelsea Dielectric Press, London 1983.

[4] J. van Turnhout, Thermally Stimulated Discharge of Polymer Electrets, Elsevier, Amsterdam 1975, Chapt. 6.

[5] J. Vanderschueren, J. Gasiot, in: Thermally Stimulated Relaxation in Solids, Topics in Applied Physics, Vol. 37, Ed. P. Bräunlich, Springer-Verlag, Berlin 1979, Chapt. 4.

[6] Thermal and Photostimulated Currents in Insulators, Ed. D.M. Smyth, The Electrochem. Soc. Inc., New Jersey 1976.

[7] J. van Turnhout, in: Electrets, Topics in Applied Physics, Vol. 33, Ed. C.M. Sessler, Springer-Verlag, Berlin-Heidelberg-New York 1980, Chapt. 3.

[8] Yu.A. Gorokhovatski, Osnovy Termodepolarizationnogo Analiza, Nauka, Moskva 1981 (in Russian).

[9] R. Chen, Y. Kirsh, Analysis of Thermally Stimulated Processes, Oxford 1981, Chapts. 1.3, 1.5, 1.9b and 3.

[10] B. Hilczer, J. Malecki, Electrets, Elsevier, Amsterdam 1986, Chapt. 6.2.

[11] V. Harašta, J. Thurzo, Fyz. Casop. (Czech.) 20, 148 (1970).

[12] K.W. Wagner, Arch. Elektrotech. II, 371 (1914). 